\title{
The falsification of Chiral Nuclear Forces
}

\author{
E. Ruiz Arriola ${ }^{1}$ a b , J. E. Amaro ${ }^{1}$, and R. Navarro Perez ${ }^{2}$ \\ ${ }^{1}$ Departamento de Física Atómica, Molecular y Nuclear and \\ Instituto Carlos I de Física Teórica y Computacional, Universidad de Granada \\ E-18071 Granada, Spain \\ ${ }^{2}$ Nuclear and Chemical Science Division, Lawrence Livermore National Laboratory \\ Livermore, California 94551, USA
}

\begin{abstract}
Predictive power in theoretical nuclear physics has been a major concern in the study of nuclear structure and reactions. The Effective Field Theory (EFT) based on chiral expansions provides a model independent hierarchy for many body forces at long distances but their predictive power may be undermined by the regularization scheme dependence induced by the counterterms and encoding the short distances dynamics which seem to dominate the uncertainties. We analyze several examples including zero energy $\mathrm{NN}$ scattering or perturbative counterterm-free peripheral scattering where one would expect these methods to work best and unveil relevant systematic discrepancies when a fair comparison to the Granada-2013 NN-database and partial wave analysis (PWA) is undertaken.
\end{abstract}

\section{Introduction}

Nuclear Physics has always been characterized by the fact that experiment is much more precise than theory. For nuclear masses one has $\Delta M(Z, N)^{\exp }<1 \mathrm{KeV} \ll \Delta M(Z, N)^{\text {th }}$ but it is unclear what the theoretical uncertainty is. Traditionally, the theoretical and reductionist predictive power flow is expected to be from light to heavy nuclei form a Hamiltonian with multinucleon forces

$$
H(A)=T+V_{2 N}+V_{3 N}+V_{4 N}+\ldots \quad \rightarrow \quad H(A) \Psi_{n}=E_{n}(A) \Psi_{n} .
$$

In the absence of $a b$ initio determinations, phenomenological $V_{n N}$ interactions are adjusted to NN scattering and light nuclei binding energies. The chiral approach, originally suggested by Weinberg in 1990 [1] (see e.g. [2-4] for reviews) to nuclear forces provides a power counting in terms of the pion weak decay constant $f_{\pi}$, with the appealing feature of systematically providing a hierarchy

$$
V_{2 N}^{\chi} \gg V_{3 N}^{\chi} \gg V_{4 N}^{\chi} \gg \ldots
$$

\footnotetext{
ae-mail: earriola@ugr.es

${ }^{\mathrm{b}}$ Speaker at XIIth Conference on Quark Confinement and the Hadron Spectrum. Work supported by Spanish Ministerio de Economia y Competitividad and European FEDER funds (grant FIS2014-59386-P), the Agencia de Innovacion y Desarrollo de Andalucia (grant No. FQM225), the U.S. Department of Energy by Lawrence Livermore National Laboratory under Contract No. DE-AC52-07NA27344, U.S. Department of Energy, Office of Science, Office of Nuclear Physics under Award No. DE-SC0008511 (NUCLEI SciDAC Collaboration)
} 
Because the pion mass is so small, chiral approaches are unambiguous at long distances via $1 \pi, 2 \pi, 3 \pi, \ldots$ exchanges for relative distances above a short distance cut-off $r_{c}, V^{n \pi}\left(r_{c}\right) \sim e^{-n r_{c} m_{\pi}}$. For instance, $\mathrm{NN}$ chiral potentials, constructed in perturbation theory, are universal and contain chiral constants $c_{1}, c_{3}, c_{4}, \ldots$ which can be related to $\pi N$ scattering [2-[4]. At long distances we have

$$
V_{N N}^{\chi}(r)=V_{N N}^{\pi}(r)+V_{N N}^{2 \pi}(r)+V_{N N}^{3 \pi}(r)+\ldots \quad r \gg r_{c},
$$

whereas they become singular at short distances

$$
V_{N N}^{\chi}(r)=\frac{a_{1}}{f_{\pi}^{2} r^{3}}+\frac{a_{2}}{f_{\pi}^{4} r^{5}}+\frac{a_{3}}{f_{\pi}^{6} r^{7}}+\ldots \quad r \ll r_{c},
$$

and some regularization must be introduced in any practical calculation. Thus, they trade the "old" model dependence for the "new" regulator dependence. What is the best theoretical accuracy we can get within "reasonable" cut-offs ? What is a reasonable cut-off ? Can the short distance piece be organized as a power counting compatible with the chiral expansion of the long distance piece ?

The huge effort which has been carried out over the last 25 years ellapsed since the seminal work of Weinberg, harvesting over 1000 citations, proves the computational feasibility of the chiral nuclear agenda requiring large scale calculations and many CPU computing hours. Here, we depart from the main streamline and wonder if chiral nuclear forces can be falsified or validated and, if yes, if they are useful for nuclear structure applications from the point of view of the predictive power.

Of course, all this has to do with proper assessment and evaluation of uncertainties of any sort and in particular in the $\mathrm{NN}$ interaction. Our original and simple estimates [5, 6] of $\Delta B^{\text {th }} / A \sim 0.5 \mathrm{MeV}$ has been upgraded in Ref. [7] to be enlarged to $\sim 2 \mathrm{MeV}$. These crude estimates are not far from the recent uncertainty analysis and order-by-order optimization of chiral nuclear interactions [8] including threebody forces where it is found $\Delta B^{\text {th }}\left({ }^{16} \mathrm{O}\right) / 16 \sim 4 \mathrm{MeV}$. Most of the uncertainty stems from the cut-off variation within a "reasonable" range, and is much worse than the ancient Weiszacker semiempirical mass formula, where $\Delta B_{\mathrm{sem}} / A \sim 0.1 \mathrm{MeV}$. If confirmed, it would be a rather pesimistic scenario for the chiral approach to nuclear structure pioneered by Weinberg. Motivated by this alarming possibility we have paid dedicated attention in the last five years to the issue of NN uncertainties [7, 9]. Here we focus on $V_{N N}^{2 \pi}$, corresponding to chiral $2 \pi$ exchange ( $\chi \mathrm{TPE}$ ).

\section{Validation and Falsification: Frequentist vs Bayesian}

From our point of view, making first a fair statistical treatment is a prerequisite to credibly aim at any precision goal in low energy nuclear physics where information is extracted by fits. We remind the fact that least squares $\chi^{2}$-fitting any (good or bad) model to some set of data is always possible and corresponds to just minimizing a distance between the predictions of the theory and the experimental measurements. How can we disentangle between true and false models?.

The well-known statistical approach, to which we stick, provides one probabilistic answer and depends on the number of data, $N_{\text {Dat }}$, the number of parameters determined from this data, $N_{\text {Par }}$, and the nature of experimental uncertainties. The natural question is: What is the probability that given the data the theory is correct ? This corresponds to the Bayesian approach which requires some $a$ priori expectations on the goodness of the theory regardless of the data and is dealt with often by augmenting the experimental $\chi_{\exp }^{2}$ with an additive theoretical contribution $\chi_{\mathrm{th}}^{2}$. However, it can be proven that when $N_{\text {Dat }} \gg N_{\text {Par }}$ one can ignore these a priori expectations since $\chi_{\exp }^{2} \sim N_{\text {Dat }} \gg \chi_{\text {th }}^{2} \sim$ $N_{\text {Par }}$ and proceed with the frequentist approach where just the opposite question is posed: what is the probability of data given the model ?. 1 In our analysis below, where we have $N_{\text {Dat }} \sim 8000$ and

\footnotetext{
${ }^{1}$ One could stay Bayesian if some relative weighting of $\chi_{\exp }^{2}$ and $\chi_{\mathrm{th}}^{2}$ is implemented (see [10 11] and references therein).
} 
$N_{\text {Par }} \sim 40$, we expect no fundamental differences. We thus simply ask: what is the probability $q$ that the the model is false ?. The p-value is $p=1-q$ and if $p$ is smaller than a predetermined confidence level we will not trust the model and we will declare it to be false. Note that 1 ) we can never be sure that the model is true and 2) any experiment can be right if errors are sufficiently large and the theory cannot be falsified. This said, $p=0.68$ when $\chi^{2} / v=1 \pm \sqrt{2 / v}$ with $v=N_{\text {Dat }}-N_{\text {Par }}$.

In general we expect discrepancies between theory and data and, ideally, if our theory is an approximation to the true theory we expect the optimal accuracy of the truncation to be comparable with the given experimental accuracy and both to be compatible within their corresponding uncertainties (see [12] for a Bayesian viewpoint). If this is or is not the case we validate or falsify the approximated theory against experiment and declare theory and experiment to be compatible or incompatible respectively. Optimal accuracy, while desirable, is not really needed to validate the theory. In the end largest errors dominate regardless of their origin; the approximated theory may be valid but inaccurate.

How should the discrepancies or residuals be interpreted ? Statistics has the obvious advantage that if we have no good reasons to suspect the theory we can test if residuals behave as, often gaussian, fluctuations and determine a confidence interval for fitting parameters within these fluctuations.

\section{Fitting and selecting data form coarse grained potentials}

The NN scattering amplitude has 5 independent complex components for any given energy, which must and can be determined from a complete set of measurements involving differential cross sections and polarization observables. From this point of view it is worth reminding that phase shifts obtained in PWA are not data by themselves unless a complete set of 10 fixed energy and angle dependent measurements have been carried out, a rare case among the bunch of existing $8000 \mathrm{np}+\mathrm{pp}$ scattering data below $350 \mathrm{MeV} \mathrm{LAB}$ energy and which corresponds to a maximal CM momentum of $p_{\mathrm{CM}}^{\max }=2 \mathrm{fm}^{-1}$. In order to intertwine all available, often incomplete and partially self-contradictory, information some energy dependence interpolation is needed. We assume a potential approach inspired by a Wilsonian point of view where we take a grid of equidistant radial "thick" points in coordinate space separated by the finite resolution given by the shortest de Broglie wavelength, $\Delta r=\hbar / p_{\mathrm{CM}}^{\max } \sim 0.6 \mathrm{fm}$ up to the radius $r_{c}=3 \mathrm{fm}$, above which charge dependent $1 \pi$ exchange gives the entire strong contribution. The counting of parameters [13] yields about 40 "thick" points, which can be represented by delta-shells (DS) [14] as originally proposed by Avilés [15]. The whole procedure needs long distance electromagnetic and relativistic contributions such as Coulomb, vacuum polarization and magnetic moments interactions. This approach allows to select the largest self-consistent existing NN database with a total of $6713 \mathrm{NN}$ scattering data driven by the coarse grained potential [16, 17] with the rewarding consequence that statistical uncertainties can confidently be propagated. 2 Precise determinations of chiral coefficients, $c_{1}, c_{3}, c_{4}$ [18, 19], the isospin breaking pion-nucleon [20, 21], and the pionnucleon-delta [7] coupling constants have been made.

\section{Chiral Fits, peripheral waves}

The questions on the cut-of $r_{c}$ raised above were answered by separating the potential as follows [19]

$$
V(r)=V_{\text {short }}(r) \theta\left(r_{c}-r\right)+V_{\text {long }}^{\chi}(r) \theta\left(r-r_{c}\right), \quad V_{\text {short }}=\frac{1}{2 \mu} \sum_{n} \lambda_{n} \delta\left(r-r_{n}\right),
$$

with $r_{n}=n \Delta r$. Several fits varying $r_{c}$ and $E_{\mathrm{LAB}}^{\max }$ were performed. The results were checked to be statistically consistent and are summarized in Table 1 It is striking that $D$-waves, nominaly N3LO and

\footnotetext{
${ }^{2}$ This resulting Granada-2013 database (http://www.ugr.es/ amaro/nndatabase/) will be used in our discussion.
} 
Table 1. Fits of chiral TPE potentials depending on the cutoff radius and the maximum fitting energy [19].

\begin{tabular}{lcccccc}
$\begin{array}{l}\text { Max } T_{\mathrm{LAB}} \\
\mathrm{MeV}\end{array}$ & $\begin{array}{c}r_{c} \\
\mathrm{fm}\end{array}$ & $\begin{array}{c}c_{1} \\
\mathrm{GeV}^{-1}\end{array}$ & $\begin{array}{c}c_{3} \\
\mathrm{GeV}^{-1}\end{array}$ & $\begin{array}{c}c_{4} \\
\mathrm{GeV}^{-1}\end{array}$ & $\begin{array}{c}\text { Highest } \\
\text { counterterm }\end{array}$ & $\chi^{2} / \nu$ \\
\hline 350 & 1.8 & $-0.4(11)$ & $-4.7(6)$ & $4.3(2)$ & $F$ & 1.08 \\
350 & 1.2 & $-9.8(2)$ & $0.3(1)$ & $2.84(5)$ & $F$ & 1.26 \\
125 & 1.8 & $-0.3(29)$ & $-5.8(16)$ & $4.2(7)$ & $D$ & 1.03 \\
125 & 1.2 & -0.92 & -3.89 & 4.31 & $P$ & 1.70 \\
125 & 1.2 & $-14.9(6)$ & $2.7(2)$ & $3.51(9)$ & $P$ & 1.05
\end{tabular}

forbidden by Weinberg chiral counting at N2LO, are indispensable !. Furthermore, data and N2LO do not support $r_{c}<1.8 \mathrm{fm}$, while several $\chi$-potentials [22, 23] take $r_{c}=0.9-1.1 \mathrm{fm}$ as "reasonable".

An alternative way of checking the failure of the power counting is provided by a deconstruction argument [19]. This corresponds to determine under what conditions are the short distance phases $\delta_{\text {short }}$, i.e. the phase shifts stemming solely from $V_{\text {short }}$ compatible with zero within uncertainties, i.e. $\left|V_{\text {short }}\right|<\Delta V$ ?. This corresponds to check what partial waves fullfill $\left|\delta_{\text {short }}\right| \leq \Delta \delta_{\text {stat }}$ when $r_{c}=1.8 \mathrm{fm}$. Unfortunately, this does not work for D-waves, supporting the previous conclusions.

The long distance character of $\chi$ TPE makes peripheral phases (large angular momentum) to be suitable for a perturbative comparison without counterterms [24 26]. However, one should take into account that 1) peripheral phases can only be obtained from a complete phase shift analyses and 2) their uncertainties are tiny [16]. The analysis of [26] just makes an eyeball comparison which looks reasonable but the agreement was not quantified. 3 We find that peripheral waves predicted by 5 thorder chiral perturbation theory are not consistent with the Granada-2013 self-consistent NN database

$$
\left|\delta^{\mathrm{Ch}, \mathrm{N} 4 \mathrm{LO}}-\delta^{\mathrm{PWA}}\right|>\Delta \delta^{\mathrm{PWA}, \text { stat }} .
$$

Sometimes we get even $3 \sigma$ discrepancies. More details on this peripheral analysis will be presented elsewhere. Of course, one may thing that $125 \mathrm{MeV}$ is too large an energy. We find that when we go down to $40 \mathrm{MeV}$, the $\chi \mathrm{TPE}$ potential becomes invisible being compatible with zero [13, 27].

The chiral potential (including $\Delta$-degrees of freedom) of Ref. [23] explicitly violates Weinberg's counting since it has N2LO long distance and N3LO short distance pieces, and residuals are not gaussian. More recently, the local short distance components of this potential have been fitted up to $125 \mathrm{MeV}$ LAB energy [28] improving the goodness of the fit, similarly to [19] (see also table 1).

\section{Chiral interactions and zero energy renormalization}

The low energy threshold parameters allow to probe the structure of chiral potentials against the NN interaction. The current approach to chiral interactions is to incorporate the $\chi$ TPE tail and include short range counterterms fitted to pp and np phase-shifts or scattering data [29, 30]. ${ }^{4}$ However, these approaches are subject to strong systematic uncertainties since a fit to phase-shifts may be subjected to off-shell ambiguities and so far low energy chiral potentials fitted to data have not achieved gaussian residuals [30] or even have huge [22] or moderate [23] $\chi^{2} / v$ values. To avoid these shortcomings we use $\chi$ TPE [13, 32] with a simpler short range structure inferred from low energy threshold parameters [7] with their uncertainties inherited from the 2013-Granada fit [16]. This corresponds to zero energy renormalization condition of the counterterms.

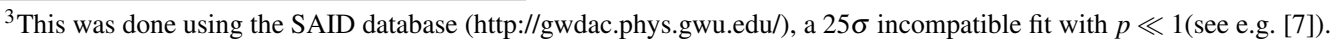

${ }^{4}$ In momentum space counterterms corresponds to coefficients of polynomials, see e.g. [31], which can be fixed by low energy threshold parameters by implicit renormalization.
} 
Table 2. Delta-Shell parameters located at $r_{1}=0.9 \mathrm{fm}$ and $r_{2}=1.8 \mathrm{fm}$ reproducing low energy threshold parameters for the indicated waves in the DS- $\chi$ TPE potential (see main text).

\begin{tabular}{ccccccccc}
\hline & ${ }^{1} S_{0}$ & ${ }^{3} P_{0}$ & ${ }^{1} P_{1}$ & ${ }^{3} P_{1}$ & ${ }^{3} S_{1}$ & $\varepsilon_{1}$ & ${ }^{3} D_{1}$ & ${ }^{3} P_{2}$ \\
\hline$\lambda_{1}$ & $-0.572(7)$ & - & - & - & $-0.368(9)$ & $-0.706(7)$ & $-4.15(1)$ & - \\
$\lambda_{2}$ & $-0.201(3)$ & $-0.033(3)$ & $0.103(7)$ & $0.221(2)$ & $-0.246(4)$ & $-0.386(7)$ & $0.35(1)$ & $-0.125(1)$ \\
\hline
\end{tabular}

One could naively expect to be able to set any number of short range counterterms to reproduce the same number of low energy threshold parameters. Actually, in order to have as the 9 counterterms dictated by Weinberg to N2LO as in [29] we need to fix $\alpha_{0}$ and $r_{0}$ for both ${ }^{1} S_{0}$ and ${ }^{3} S_{1}$ waves, the mixing $\alpha_{\varepsilon}$ and $\alpha_{1}$ for the ${ }^{3} P_{0},{ }^{3} P_{1},{ }^{3} P_{2},{ }^{1} P_{1}$ [7]. In practice this turned out to be unfeasible in particular for the $J=1$ coupled channel where one has matrices $\mathbf{a}$ and $\mathbf{r}_{0}$. If instead one includes two counterterms in each partial wave in the $J=1$ coupled channel it is then possible to reproduce the coupled channel $\mathbf{a}$ and $\mathbf{r}_{0}$ matrices. With this structure we have a total of 12 short range parameters set to reproduce 12 low energy threshold parameters from [7], and not the 9 expected from N2LO [29]. Statistical uncertainties can be propagated by making fits to each of the 1020 sets of threshold parameters that were calculated from the bootstrap generated DS potentials [33]; this directly takes into account any statistical correlation between low energy parameters. Table 2 lists the resulting $12-\lambda_{i}$ parameters. In Figure 1 we show the phase-shifts corresponding to the DS- $\chi$ TPE potential with the parameters of Table 2 and compare them to the DS-OPE potential [16, 34]. We observe a good agreement between both representations up to a laboratory energy of $20 \mathrm{MeV}$.
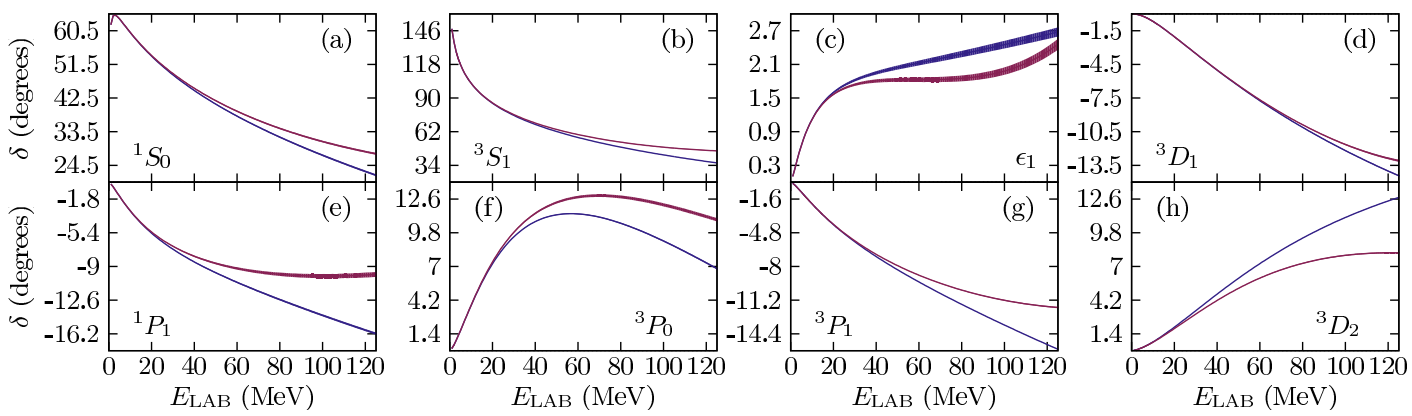

Figure 1. $\chi$ TPE zero energy renormalized np phase-shifts fixing the low energy threshold parameters (see main text) [7] compared with the phases obtained from the fit to the 2013-Granada database [16].

\section{Conclusions}

Chiral nuclear forces have been massively implemented in Nuclear Physics in the last 25 years with the legitimate hope of providing a unified description of nuclear phenomena more rooted in QCD and less model dependent than most of the phenomenological approaches. This huge effort proves that they are not only calculable but also that they can be used in light nuclei studies, but their indispensability remains to be established. Their systematic uncertainties may be large and they might not be necessarily more predictive than the usual phenomenological and non-chiral approaches. Within the EFT approach there is a residual model dependence regarding the finite cut-off regularization 
scheme, which seems to dominate the uncertainties. Therefore, efforts should be placed on reducing this largest source of error. We stress that none of these results invalidates $\chi \mathrm{TPE}$ above $r_{c}=1.8 \mathrm{fm}$, but it does question the status of Weinberg's power counting encoding short distance ignorance.

\section{References}

[1] S. Weinberg, Phys. Lett. B251, 288 (1990)

[2] P.F. Bedaque, U. van Kolck, Ann. Rev. Nucl. Part. Sci. 52, 339 (2002), nucl-th/0203055

[3] E. Epelbaum, H.W. Hammer, U.G. Meissner, Rev. Mod. Phys. 81, 1773 (2009), 0811. 1338

[4] R. Machleidt, D. Entem, Phys.Rept. 503, 1 (2011), 1105. 2919

[5] R. Navarro Pérez, J.E. Amaro, E. Ruiz Arriola (2012), 1202 . 6624

[6] R. Navarro Pérez, J.E. Amaro, E. Ruiz Arriola, PoS QNP2012, 145 (2012), 1206.3508

[7] R.N. Perez, J.E. Amaro, E. Ruiz Arriola, J. Phys. G43, 114001 (2016), 1410.8097

[8] B.D. Carlsson, A. Ekström, C. Forssén, D.F. Strömberg, G.R. Jansen, O. Lilja, M. Lindby, B.A. Mattsson, K.A. Wendt, Phys. Rev. X6, 011019 (2016), 1506.02466

[9] R. Navarro Pérez, J.E. Amaro, E. Ruiz Arriola, J. Phys. G42, 034013 (2015), 1406.0625

[10] T. Ledwig, J. Nieves, A. Pich, E. Ruiz Arriola, J. Ruiz de Elvira, Phys. Rev. D90, 114020 (2014)

[11] R.N. Perez, J.E. Amaro, E. Ruiz Arriola, Int. J. Mod. Phys. E25, 1641009 (2016), 1601.08220

[12] S. Wesolowski, N. Klco, R.J. Furnstahl, D.R. Phillips, A. Thapaliya, J. Phys. G43,074001(2016)

[13] R. Navarro Pérez, J.E. Amaro, E. Ruiz Arriola, Few-Body Systems pp. 1-5 (2014), 1310. 8167

[14] R. Navarro Pérez, J.E. Amaro, E. Ruiz Arriola, Phys.Lett. B724, 138 (2013), 1202 . 2689

[15] J. Aviles, Phys.Rev. C6, 1467 (1972)

[16] R. Navarro Pérez, J.E. Amaro, E. Ruiz Arriola, Phys.Rev. C88, 064002 (2013), 1310. 2536

[17] R. Navarro Pérez, J.E. Amaro, E. Ruiz Arriola, Phys.Rev. C89, 064006 (2014), 1404 .0314

[18] R. Navarro Pérez, J.E. Amaro, E. Ruiz Arriola, Phys. Rev. C91, 054002 (2015), 1411.1212

[19] R. Navarro Pérez, J.E. Amaro, E. Ruiz Arriola, PoS CD12, 104 (2013), 1301.6949

[20] R.N. Perez, J.E. Amaro, E. Ruiz Arriola (2016), 1606.00592

[21] E. Ruiz Arriola, J.E. Amaro, R. Navarro Pérez, Mod. Phys. Lett. A31, 1630027 (2016)

[22] A. Gezerlis, I. Tews, E. Epelbaum, M. Freunek, S. Gandolfi, K. Hebeler, A. Nogga, A. Schwenk, Phys. Rev. C90, 054323 (2014), 1406.0454

[23] M. Piarulli, L. Girlanda, R. Schiavilla, R. Navarro Pérez, J.E. Amaro, E. Ruiz Arriola, Phys. Rev. C91, 024003 (2015), 1412.6446

[24] N. Kaiser, R. Brockmann, W. Weise, Nucl. Phys. A625, 758 (1997), nucl-th/9706045

[25] N. Kaiser, S. Gerstendorfer, W. Weise, Nucl. Phys. A637, 395 (1998), nucl-th/9802071

[26] D.R. Entem, N. Kaiser, R. Machleidt, Y. Nosyk, Phys. Rev. C91, 014002 (2015), 1411.5335

[27] J.E. Amaro, R. Navarro Pérez, E. Ruiz Arriola, Few-Body Systems pp. 1-5 (2013), 1310. 7456

[28] M. Piarulli, L. Girlanda, R. Schiavilla, A. Kievsky, A. Lovato, L.E. Marcucci, S.C. Pieper, M. Viviani, R.B. Wiringa (2016), 1606.06335

[29] A. Ekström et al., Phys. Rev. Lett. 110, 192502 (2013), 1303.4674

[30] A. Ekström, B.D. Carlsson, K.A. Wendt, C. Forssén, M. Hjorth-Jensen, R. Machleidt, S.M. Wild, J. Phys. G42, 034003 (2015)

[31] E. Ruiz Arriola, Symmetry 8, 42 (2016)

[32] R. Navarro Pérez, J.E. Amaro, E. Ruiz Arriola, Phys.Rev. C89, 024004 (2014), 1310. 6972

[33] R. Navarro Perez, J. Amaro, E. Ruiz Arriola, Phys.Lett. B738, 155 (2014), 1407 . 3937

[34] R. Navarro Pérez, J.E. Amaro, E. Ruiz Arriola, Phys.Rev. C88, 024002 (2013), 1304.0895 